\title{
Cardiac fibroma mimicking hypertrophic cardiomyopathy in a 5-month-old infant
}

\author{
Feifei Sun ${ }^{1}$, Fangfang $\mathrm{Li}^{1}$, Liping Huang ${ }^{1}$, Weidong Ren ${ }^{1 *}$ and Xin Zhang ${ }^{2}$ \\ ${ }^{1}$ Department of Ultrasound, Shengiing Hospital of China Medical University, Shenyang, China \\ ${ }^{2}$ Department of Radiology, the First Affiliated Hospital of China Medical University, Shenyang, China
}

\begin{abstract}
Primary cardiac tumors in children are rare. Occasionally, they can mimic hypertrophic cardiomyopathy on echocardiography. We present a primary cardiac fibroma in a 5-month-old boy that mimicked hypertrophic cardiomyopathy according to echocardiography and cardiac magnetic resonance. We diagnosed the cardiac tumor at a young age and observed the case with more than 5-years of follow-up, which showed no obvious change in clinical status and echocardiographic features.
\end{abstract}

\section{Introduction}

Primary cardiac tumors in children are rare; that is, only 0.027 $0.08 \%$. Nearly $75 \%$ of cardiac tumors are benign, with fibroma accounting for approximately $25 \%[1,2]$. The symptoms vary from absent to nonspecific, and this usually causes a delayed diagnosis and surgery [3]. Here, we present a primary cardiac fibroma in a very young patient that mimicked hypertrophic cardiomyopathy.

\section{Case report}

A 5-month-old boy was admitted to the pediatric cardiac department of our hospital because of fever and palpitations. He had a biological sister who was 8-years-old and healthy. Electrocardiogram (ECG) showed atrial arrhythmias (atrial premature and atrial fibrillation) associated with $\mathrm{T}$ wave inversion from $\mathrm{V} 1$ through $\mathrm{V} 3$ and a low-flat in leads V4-V6 (Figure 1A). Two-dimensional transthoracic echocardiography (2D TTE) of the parasternal left ventricular shortaxis section revealed an obvious asymmetric hypertrophy of the medium-posterior interventricular septum (maximal septal thickness $=13 \mathrm{~mm}$; septal-free wall ratio $=4.3$; Figure 1B). A 2D TTE parasternal 4-chamber section showed the thickened interventricular septum was localized to the basal region (Figure 1C). However, the ultrasonic echo of the thickened part was obviously higher than that of the normal myocardial tissue. Myocardial thickening and echogenicity, just like an egg yolk contained in an egg, was observed with deformation during the cardiac cycle (Video 1). This heterogeneous echogenicity raised the suspicion of a cardiac mass rather than ordinary hypertrophic cardiomyopathy. There was neither outflow tract obstruction nor stenosis of the valvular annulus (Video 2). There was distortion of the left ventricle, and 2D and Doppler revealed mild anterior mitral valve prolapse and mild mitral regurgitation. The cardiac function of the patient was normal. To provide a more accurate evaluation of the local interventricular septum hypertrophy and tissue characterization, cardiac magnetic resonance imaging (MRI) using a 1.5 Tesla scanner was subsequently performed. Because the patient was very young, and his kidney function was incapable of gadolinium, a cardiac MRI was performed without contrast enhanced imaging. A cine (steady-state free precession) view of the MRI confirmed the regional myocardial thickening (Figure 1D), which had signal characteristics similar to the remaining myocardium on T1-weighted fast spin-echo and fat suppression images (Figure 1E). Moreover, it appeared with mild hypo-intensity on T2-weighted fast spin-echo images (Figure 1F). These typical MRI characterizations permitted ruling out the presence of hypertrophic cardiomyopathy and allowed a confident diagnosis of rare cardiac fibroma (Videos 3 and 4). Surgical resection was deemed not necessary at the time because of the absence of clinical indications (i.e., severe arrhythmias, hemodynamic or respiratory compromise, or a significant risk of systemic embolic events) [4]. In addition, the patient had endomyocardial fibro elastosis syndrome when he was 1-year-old, which was cured. He was also found to have a black verrucous nevus in the facial, neck, and trunk areas from birth. Oral antiarrhythmic agent therapy was continued under close echocardiographic and rhythmic monitoring with follow-up evaluation at 5 years. During these years, echocardiographic features of the patient in this case were unchanged and clinical symptoms of the heart were stable.

\section{Discussion}

Cardiac fibromas are rare, congenital, histologically benign tumors usuallylocated in theleft ventricular free wall or septum [5]. Occasionally, as described in this case, they can mimic hypertrophic cardiomyopathy on echocardiography. In these cases, differential diagnosis is crucial. The infant in this case had the symptoms of arrhythmia and $\mathrm{T}$ wave inversion on ECG, which we initially suspected heart disease. Then, the hypertrophy of the regional interventricular septum raised the suspicion of a cardiac mass due to the echocardiographic features of hyperreflectivity and heterogeneous echogenicity. Moreover, the thickened myocardium showed passive motion along with the remaining myocardium. Hypertrophic cardiomyopathy is the most common

Correspondence to: Weidong Ren, Department of Ultrasound, Shengjing Hospital of China Medical University, Sanhao Street, Heping District, Shenyang, China, Tel: +86-24-96615-74191; Fax: +86-24-64679608; E-mail: rwd0410@163.com

Key words: cardiac tumor, congenital heart defect, echocardiography, cardiac magnetic resonance

Received: November 22, 2017; Accepted: December 23, 2017; Published: December 26, 2017 

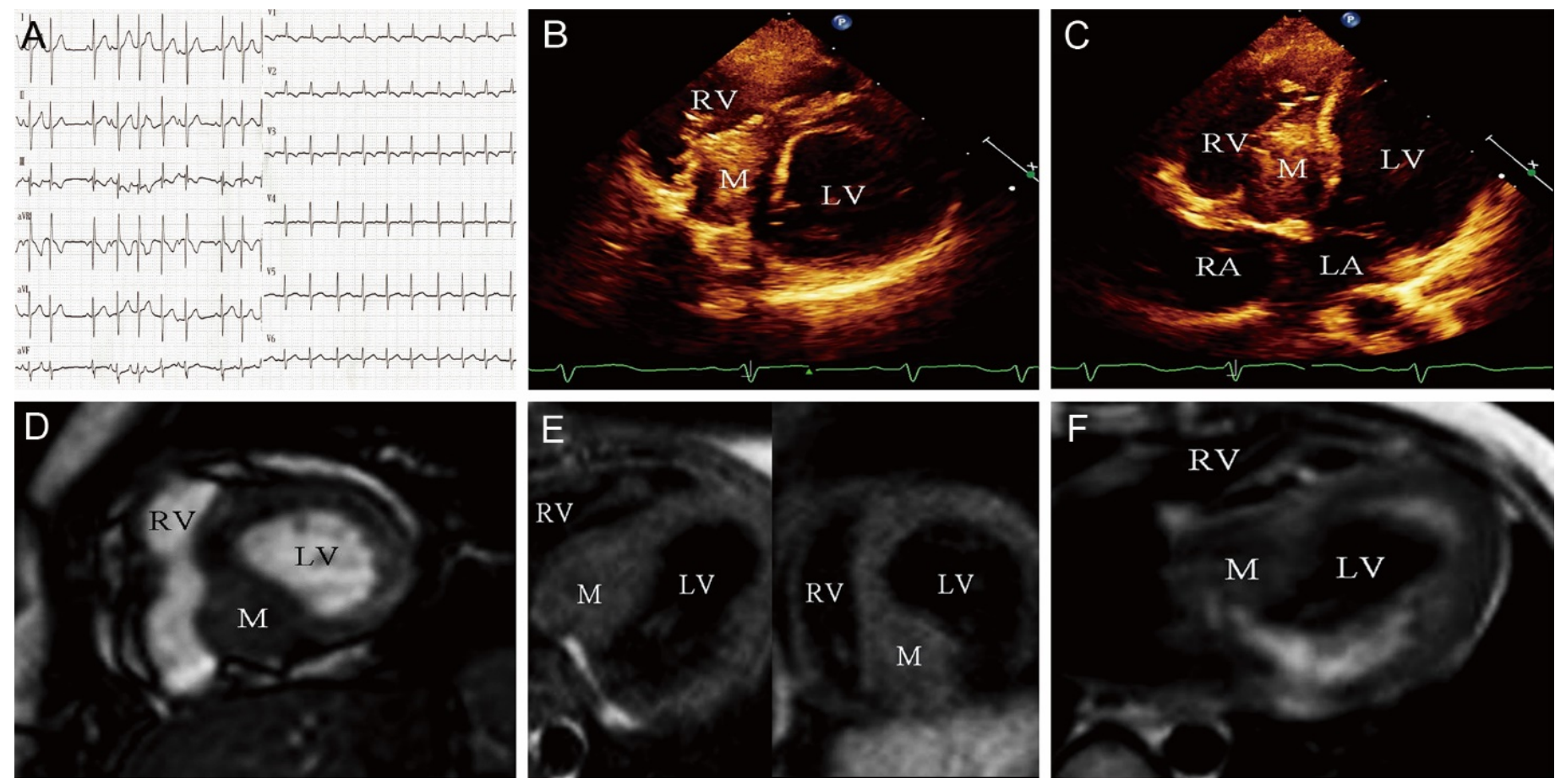

Figure 1. Primary cardiac fibroma in a 5-month-old infant.

(A) Electrocardiogram showing the atrial arrhythmias associated with $\mathrm{T}$ wave changes.

(B) A two-dimensional transthoracic echocardiography (2D TTE) left ventricle short-axis view revealed local asymmetric hypertrophy in the medium-posterior interventricular septum, presenting with an image of the mass.

(C) 2D TTE 4-chamber view showed the distinct mass-like local thickening in a basal segment of the interventricular septum. Cardiac magnetic resonance imaging (MRI) confirmed regional myocardial thickening.

(D)Cardiac magnetic resonance imaging (MRI) confirmed regional myocardial thickening.

(E) MRI showing signal characteristics similar to the remaining myocardium in T1-weighted fast spin-echo (left) and fat suppression images (right).

(F) MRI demonstrated slightly hypo-intensity on T2-weighted fast spin-echo images. LA indicates left atrium; LV, left ventricle; M, mass; RA, right atrium; and RV, right ventricle.

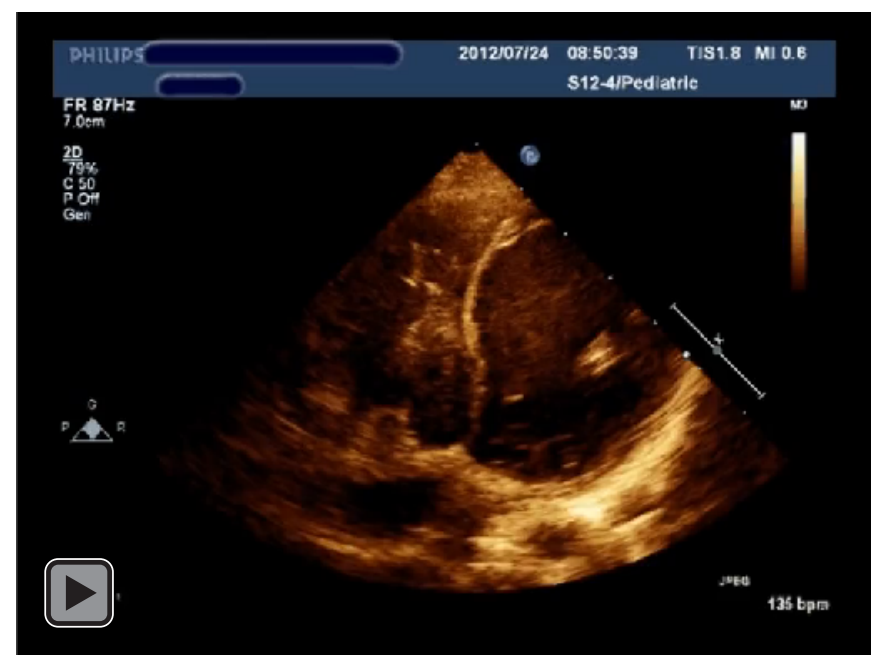

Video 1. 2D TTE showing asymmetric hypertrophy of the interventricular septum and an intramyocardial tumor with deformation in the cardiac cycle.

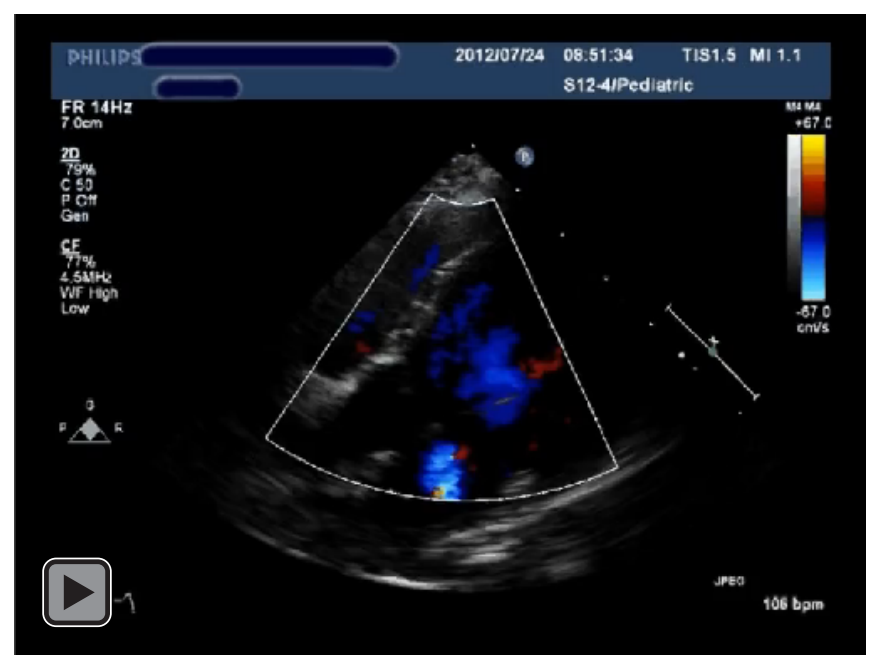

Video 2. 2D TTE parasternal 4-chamber section color Doppler view demonstrated no left ventricle outflow and inflow tract obstruction. 


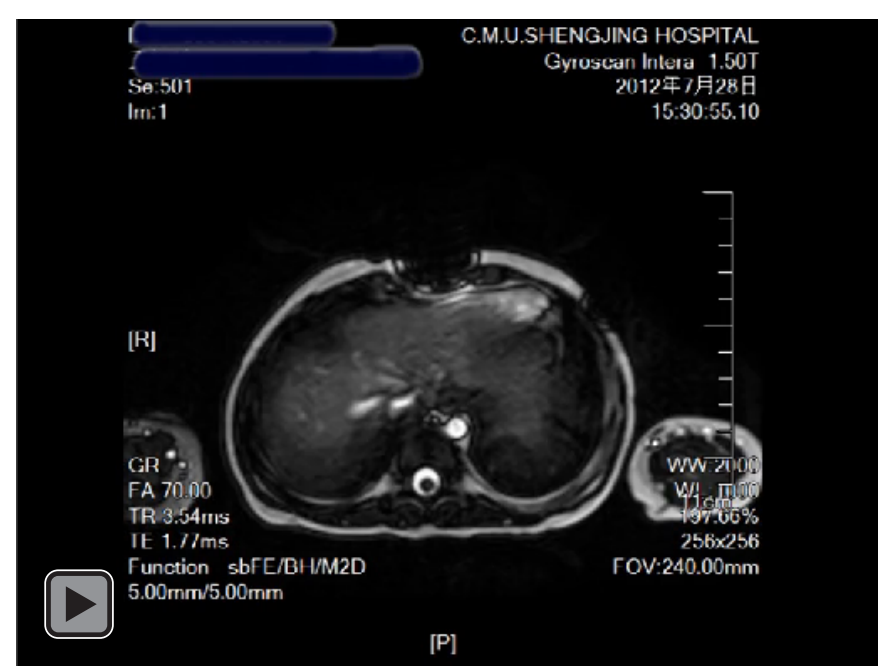

Video 3. Cine view of MRI axial imaging confirming the regional myocardial thickening.

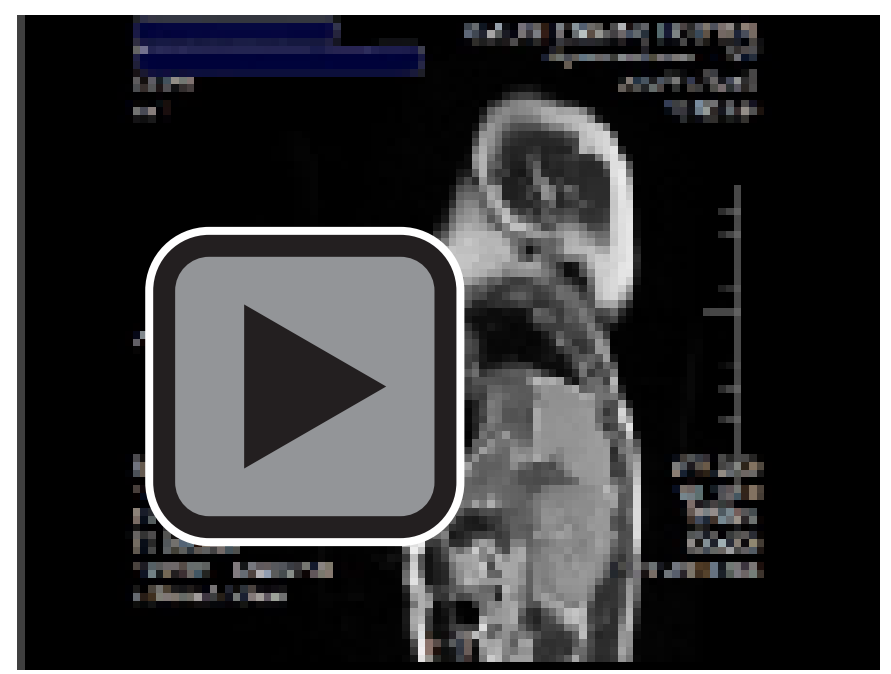

Video 4. Cine view of MRI sagittal imaging shows regional hypertrophy of the interventricular septum.

cause of ventricular septal regional thickening, and it can mimic the symptoms of an intramural cardiac tumor through the cardiac MRI with T1 iso-intensity, T2 slightly hypo-intensity and no signal change after fat suppression imaging, which were the characteristics of fibroma due to its fibrous composition and low water content.

Fibroma is considered as the second most common primary cardiac intramural tumor in children, while rhabdomyoma is the first [6]. Echocardiographic and MRI features can provide an important reference for differentiating the above-mentioned tumors. Fibromas are typically solitary, hyper refractile, well-demarcated, intramural left ventricular masses, mostly with a low tendency to recur and grow aggressively. They show a high possibility of affecting the conduction system, which results in arrhythmia, conduction blocking and ventricular tachycardia that may cause sudden death, which occurs in one-third of the patients [3]. A study has shown that a fibroma located in the septal regions is a significant prognostic factor due to its high tendency for arrhythmias [7]. It may block the outflow or inflow tracts, influencing the hemodynamics, which results in chest pain, shortness of breath or even heart failure. Many studies have stressed the crucial role of echocardiography as it is non-invasive, convenient and has sensitivity for analyzing small tumors and those located on valves and shows the tumor size, location, and the hemodynamics and functions of the heart [8]. Therefore, echocardiography today is considered as routine practice. Computed tomography and MRI are required as secondlevel examinations to determine the location and relationship with the surrounding structures as they are essential for surgeries [9]. There are obvious differences in MRI image characteristics between myocardial fibroma and rhabdomyoma [1]. For fibroma, the characteristics include: 1) intramyocardial locations involving the ventricular septum or free wall; 2) well-defined borders with a thin rim of the myocardium; and 3) a heterogeneous appearance on T1- and T2-weighted TSE sequences with T1 iso-intensity, T2 slightly hypo-intensity and no signal change after fat suppression imaging. For rhabdomyoma, the characteristics include: 1) intramyocardial or intracavitary locations attached to the myocardium; 2) mildly hyperintense on T2-weighted TSE imaging; and 3) homogenous appearance on all sequences.

The significance of this case is that we diagnosed the cardiac tumor at a young age and observed the case with more than 5-years of follow-up, which showed no obvious change in clinical status and echocardiographic features. The literature shows that younger fibroma patients may have a poorer outcome compared to older children, which obviously shows the importance of early diagnosis [6]. Resection operations of fibromas have typically good postoperative prognoses, while surveillance imaging should be performed in the case of recurrence. Since the patient showed absence of clinical indications, in addition to the young age, we recommended long-term follow up instead of surgery. For a patient with obvious symptoms, a resection of the tumor to release the symptoms and prevent further complications and risk of sudden death should be considered since there is a high probability of arrhythmias and ventricular tachycardia [4]. Echocardiography and MRI are sensitive for the diagnosis of fibromas. Although in occasional cases a differential diagnosis may be difficult, careful attention to the echocardiographic and/or MRI features should lead to the correct diagnosis in most cases.

\section{References}

1. Miyake CY, Del Nido PJ, Alexander ME, Cecchin F, Berul CI, et al. (2011) Cardiac tumors and associated arrhythmias in pediatric patients, with observations on surgical therapy for ventricular tachycardia. J Am Coll Cardiol 2011; 58: 1903-1909. [Crossref]

2. Beroukhim RS, Prakash A, Buechel ER, Cava JR, Dorfman AL, et al. (2011) Characterization of cardiac tumors in children by cardiovascular magnetic resonance imaging: a multicenter experience. J Am Coll Cardiol 58: 1044-1054. [Crossref]

3. Neragi-Miandoab S, Kim J, Vlahakes GJ (2007) Malignant tumours of the heart: a review of tumour type, diagnosis and therapy. Clin Oncol (R Coll Radiol) 19: 748-756. [Crossref]

4. Stiller B, Hetzer R, Meyer R, Dittrich S, Pees C, et al. (2001) Primary cardiac tumours: when is surgery necessary? Eur J Cardiothorac Surg 20: 1002-1006. [Crossref]

5. Syed IS1, Feng D, Harris SR, Martinez MW, Misselt AJ, et al. (2008) MR imaging of cardiac masses. Magn Reson Imaging Clin N Am 16: 137-164. [Crossref]

6. Jha NK, Kiraly L, Tamas C, Talo H, Khan MD, et al. (2015) Large cardiac fibroma and teratoma in children- case reports. J Cardiothorac Surg 10: 38. [Crossref]

7. Torimitsu S, Nemoto T, Wakayama M, Okubo Y, Yokose T, et al. (2012) Literature survey on epidemiology and pathology of cardiac fibroma. Eur J Med Res 17: 5. [Crossref]

8. Sparrow PJ, Kurian JB, Jones TR, Sivananthan MU (2005) MR imaging of cardiac tumors. Radiographics 25: 1255-1276. [Crossref]

9. Padalino MA, Vida VL, Boccuzzo G, Tonello M, Sarris GE, et al. (2012) Surgery for primary cardiac tumors in children: early and late results in a multicenter European congenital heart surgeon's association study. Circulation 126: 22-30. [Crossref]

Copyright: (C2017 Sun F. This is an open-access article distributed under the terms of the Creative Commons Attribution License, which permits unrestricted use, distribution, and reproduction in any medium, provided the original author and source are credited. 